\title{
ВЛИЯНИЕ ОТДЕЛЬНЫХ ФАКТОРОВ НА ВЕЛИЧИНУ ОСАДКИ ПРОЕКТИРУЕМЫХ ФУНДАМЕНТОВ
}

\section{П. С. Пойта', П. В. Шведовский', Д. Н. Клебанюк ${ }^{3}$ А. А. Четырбок ${ }^{4}$}

\author{
1 Д. т. н., профрессор, профрессор кафедры геотехники и транспортных коммуникаций \\ учреждения образования «Брестский государственньй технический университет», Брест, Беларусь, e-mail: ofig@bstu.by \\ ${ }^{2}$ K. т. н., профрессор, профессор кафредры геотехники и транспортных коммуникаций \\ учреждения образования «Брестский государственный технический университет», Брест, Беларусь, e-mail: ofig@bstu.by \\ ${ }^{3}$ Магистр технических наук, старший преподаватель кафедры геотехники и транспортных коммуникаций \\ учреждения образования «Брестский государственный технический универсuтет», Брест, Беларусь, e-mail: klebanyuk.dmitri@gmail.com \\ ${ }^{4}$ Студентка строительного фракультета гр. А44, 3 курс \\ учреждения образования «Брестский государственный технический университет», Брест, Беларусь
}

\begin{abstract}
Реферат
При постоянном давлении осадки квадратных (круглых) фундаментов чаще всего меньше в сравнении с ленточными при прочих равных условиях. При переходе от квадратных и круглых фрундаментов к прямоугольным и ленточным и росте коэффициента отношения сторон увеличивается возможность поперечного вытеснения грунта из-под фундамента и соответственно приводит к развитию больших осадок.

В данной статье представлены результаты исследования по поиску решений, обеспечивающих минимизацию величин осадок зданий и сооружения и их неравномерность. Выявлено, что применение квадратных (круглых) фундаментов позволяет не только уменьшить величину осадки, но и избежать большой ее неравномерности.
\end{abstract}

Ключевые слова: нагрузка, давление, осадка, фундамент, площадь, грунты, деформации, коэффициента отношения сторон.

\section{INFLUENCE OF INDIVIDUAL FACTORS ON THE VALUE OF SETTLEMENT OF DESIGNED FOUNDATIONS}

\section{Abstract}

\section{P. S. Poyta, P. V. Shvedovsky, D. N. Klebanyuk, A. A. Chetyrbok}

At a constant pressure, the settlement of square (round foundations) foundations is less than that of strip foundations, other conditions being equal. The transition from square and circular foundations to rectangular and strip foundations and the increase in the aspect ratio increases the possibility of transverse displacement of soil from under the foundation and correspondingly leads to the development of high settlement values.

This paper presents the results of the research to find solutions which ensure minimization of building and structure settlements and their nonuniformity. It is revealed that the use of compact foundations makes it possible not only to reduce the value of settlement, but also to avoid its large irregularity.

Keywords: load, pressure, settlement, foundation, area, soils, deformation, aspect ratio.

\section{Введение}

Под действием нагрузок от зданий и сооружений основания фундаментов претерпевают деформации, внешним проявлением которых являются осадки (равномерные и неравномерные), горизонтальные смещения и крены. Равномерные осадки не вызывают в фундаментах и вышерасположенных конструкциях дополнительных усилий и не нарушают их нормальную эксплуатацию. Они не влияют и на их прочность, хотя чрезмерно большие значения осадок вызывают ряд проблем с точки зрения нормальной эксплуатационной пригодности зданий и сооружений.

При строительстве на сжимаемых грунтах разность осадок отдельных фундаментов достигает значительных величин и в зданиях если не предусмотреть специальных мер, появляются опасные деформации, иногда приводящие их в состояние, непригодное для безопасной эксплуатации.

В связи с этим, при проектировании фундаментов следует стремиться к максимальному выравниванию осадок с тем, чтобы их разность не превышала установленных нормами пределов.

Определенные резервы для этого, на наш взгляд, заложены в выборе рациональной формы подошвы, ее площади и глубины заложения.

Влияние формы подошвы фундамента на конечную осадку здания

Полная конечная осадка фундамента зависит от целого ряда факторов, основными из которых являются: величина напряжений в основании, вызванная передаваемой нагрузкой и загружением прилегающих площадей; размеры, форма, жесткость и глубина заложения фундамента; величина пластических зон под краями фундамента; мощность сжимаемой толщи; свойства слоев грунта, слагающих основание (характеристика деформируемости, уплотненность и т. д.) и его распределительная способность; степень нарушения естественных условий залегания грунтов при производстве работ [1] Из всего многообразия факторов, влияющих на осадку фундамента, рассмотрим основные, обычно учитываемые при проектировании фундаментов, но, как нам кажется, не всегда в полной мере оцененные. К таким фракторам можно отнести форму фундамента, его размеры, глубину заложения и грунтовые условия.

Рассмотрим влияние размеров фундамента на его осадку $S$ В соответствии с классическим решением теории упругости

$$
S=\frac{\omega \cdot P \cdot\left(1-v^{2}\right) \cdot \sqrt{A}}{E^{(e)}},
$$

где $\omega$ - коэффициент, зависящий от формы подошвы (отношение сторон $\eta=I /$ b) и жесткости фундамента;

I и $b$-соответственно, длина и ширина фундамента;

$E$ и $v$ - соответственно, модуль сжатия и коэфффициент Пуассона;

$P$ - среднее давление по подошве фундамента;

$A$ - площадь фундамента.

Из уравнения (1) следует, что величина осадки (S) линейно зависит от $\sqrt{A}$. Это означает, что с увеличением линейных размеров фундамента его осадка непрерывно должна возрастать. Это можно объяснить тем обстоятельством, что увеличение размеров фундамента, как правило, связано с ростом общей нагрузки на него.

Важно отметить, что опыты в натурных условиях по изучению осадок фундаментов с площадями от 0,5 до $15 \mathrm{~m}^{2}$ на однородном 
Вестник Брестского государственного технического университета. 2021

водонасыщенном песке мощностью до 12,0 м [2], а также опыты Фундаментстроя [3] с площадями от 0,25 до 8,0 м², в пределах линейной зависимости между давлением и осадкой, позволили получить следующую эмпирическую зависимость:

$$
S=K \cdot P \cdot \sqrt{A}
$$

где $K$ - коэффицциент пропорциональности.

Однако, на основании ряда опытов [2, 3], было установлено, что при постоянном давлении и прочих равных условиях в однородных грунтах зависимость между осадкой и площадью фундамента является более сложной (рис. 1).

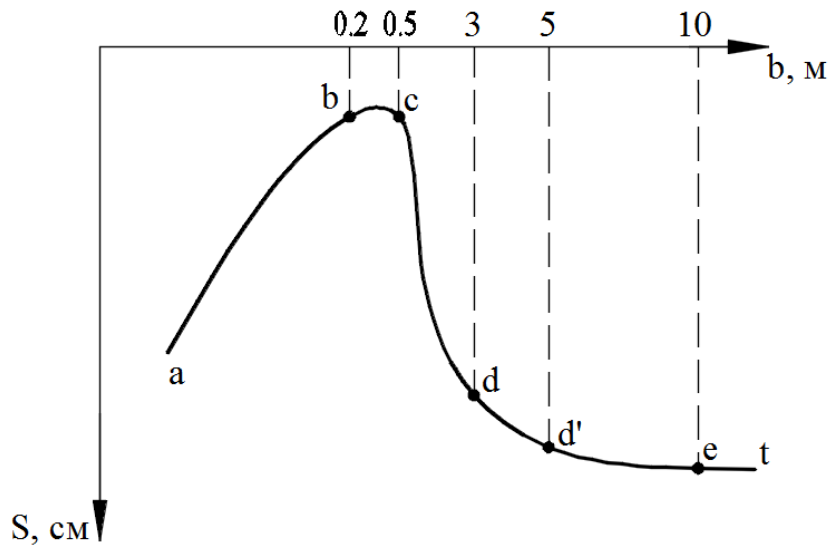

Рисунок 1 - Зависимость осадки жесткого квадратного фундамента от его ширины $S=f(b)$ при постоянном давлении

Из рис. 1 следует, что в обычно используемом диапазоне давлений на основания $0,1 \ldots 0,5$ МПа линейная зависимость $S=f(b)$ соблюдается только в пределах размеров стороны фундамента от 0,5...0,7 м до 3,0...5,0 м (до 5,0...7,0 м по Н.А.Цытовичу) [4]. Далее рост осадки замедляется и при ширине фундамента превышающей 10 м, кривая $S=f(b)$ переходит практически в горизонтальную прямую, т. е. осадка фундамента становится не зависящей от ширины фундамента.

Нами выполнены расчеты величины осадки для круглых, квадратных, прямоугольных и ленточных фундаментов. Грунтовые условия во всех вариантах расчета приняты одинаковыми. Глубина заложения фундаментов $-2,0$ м, давление $P-\sigma_{z q \cdot 0}=190 \mathrm{\kappa Па,}$ $E=10,0 \mathrm{M \Pi а}$.

На рис. 2 представлены графики влияния ширины фундамента различной формы на его осадку. В пределах изменения ширины фундамента от 0,8 м до 5,5 м, при увеличении ширины фундамента, пропорционально растет и его осадка. При этом, при одной и той же ширине подошвы осадки фундаментов квадратных и круглых значительно меньше, чем ленточных.

Так, если ширина квадратного (круглого) фундамента $b=1,0$ м, то его осадка в 2,15 раза меньше, чем осадка такого по ширине ленточного фундамента. При $b=5,0$ м (квадрат, круг) это различие меньше и составляет 1,67 раза.

Натурные и лотковые опыты, проведенные рядом исследователей $[2,4,5]$, показывают, что осадки фундаментов зависят от плотности испытуемых грунтов и, как правило, их величины у фундаментов квадратной (круглой) формы превышают осадки удлиненных фундаментов (при одинаковых площадях и давлениях). Однако такое соотношение сохраняется только в первой фазе деформации составляющая осадки за счет уплотнения грунта [1]

Более того, из опытов Muhsa [6] следует, что осадки прямоугольных фундаментов оказались больше осадок квадратных фундаментов во всех фазах деформации. Таким образом, при переходе от круглых и квадратных фундаментов к ленточным и прямоугольным (при одинаковых площадях и давлениях) увеличивается возможность поперечного вытеснения грунта из-под фундамента и, соответственно, больших осадок.

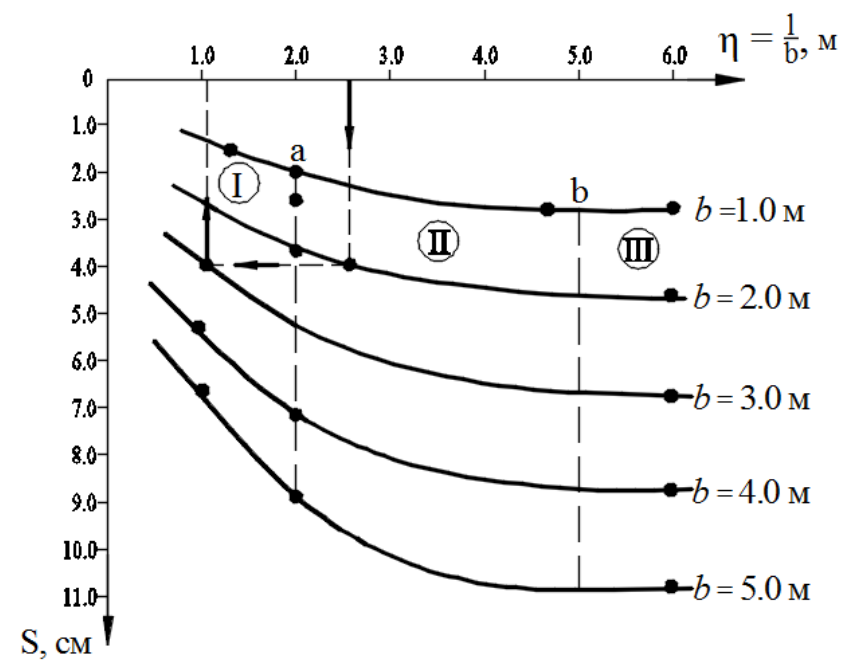

1 - фундамент квадратный (круглый), $\eta=1,0$;

2 - фундамент ленточный $\eta=\frac{l}{b} \geq 10$;

3 - фундамент прямоугольный при $\eta=\frac{l}{b}=2,0$;

4 - фундамент прямоугольный, $\eta=\frac{l}{b}=1,3$

Рисунок 2 - Зависимость осадки фундамента от его ширины

Уменьшение осадок круглых и квадратных фундаментов по сравнению с прямоугольными и ленточными при одинаковой ширине связано с более быстрым затуханием напряжений по глубине. Из рис. 2 также следует, что осадки прямоугольных фундаментов при различных значения коэффициента отношения сторон прямоугольных фундаментов $\eta=\frac{l}{b}$ (I- длина; $b$ - ширина фундамента $)$ занимают промежуточное значение между квадратными (круглыми) и ленточными фундаментами.

Весьма показательными являются зависимости $S=f(A)$, представленные на рис. 3. Их характер аналогичен кривой, представленной на рис. 1.

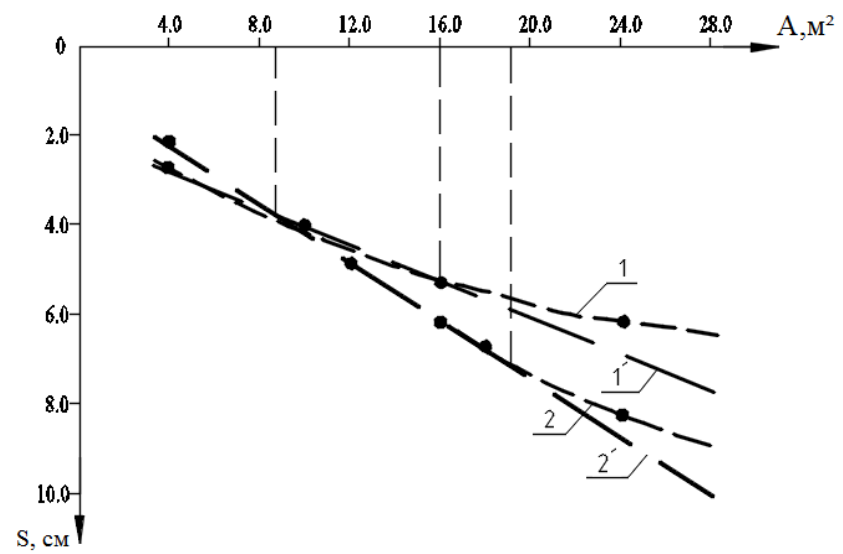

1 - фундаменты квадратные (круглые);

2 - фундаменты ленточные;

1', 2' - касательные к кривым 1 и 2

Рисунок 3 - Влияние размеров площади подошвы фундамента на его осадку при постоянном давлении 
Для квадратных фундаментов линейная зависимость соблюдается только в пределах размеров стороны фундамента от $0,6 \ldots 0,7 \mathrm{~m}$ до $3,7 \ldots 4,2$ м. Для ленточного фундамента этот предел несколько больше и составляет от $0,6 \ldots 0,7$ м до 4,0...4,4 м. Далее рост осадки замедляется и сохраняется тенденция к потере зависимости от площади фундамента.

Отличительной особенностью полученных кривых является начальный участок в пределах изменения площади фундаментов от 2,0 м² до 9,0 м²: на этом участке осадка ленточного фундамента меньше осадки более квадратного. При переходе от квадратного фундамента к ленточному (прямоугольному), при одинаковых давлении и площади, вследствие уменьшения ширины уменьшается мощность активной зоны. А это вызывает соответствующее уменьшение осадки, поскольку работу грунта основания следует рассматривать в первой фазе деформации, когда осадка происходит, в основном, за счет уплотнения грунта.

На рис. 4 показано влияние коэфффициента отношения сторон $\eta=\frac{l}{b}$ на величину осадки фундаментов при меняющейся их ширине. В общем случае на полученных графиках можно выделить три зоны. Первая - когда $\eta$ меняется от 1,0 до 2,0. На этом участке зависимость $S=f(\eta)$ прямо пропорциональная и с ростом коэфффициента $\eta$ осадки фундаментов возрастают.

Следовательно, именно при указанных выше пределах изменения $\eta$ управление осадками фундаментов различной ширинь наиболее эффективно. В зоне II происходит затухание осадки с увеличением коэффициента отношения сторон. И в третьей зоне, т. е. при $\eta=5,0$ и более, рост коэффрициента отношения сторон практически не оказывает никакого влияния на развитие осадок.

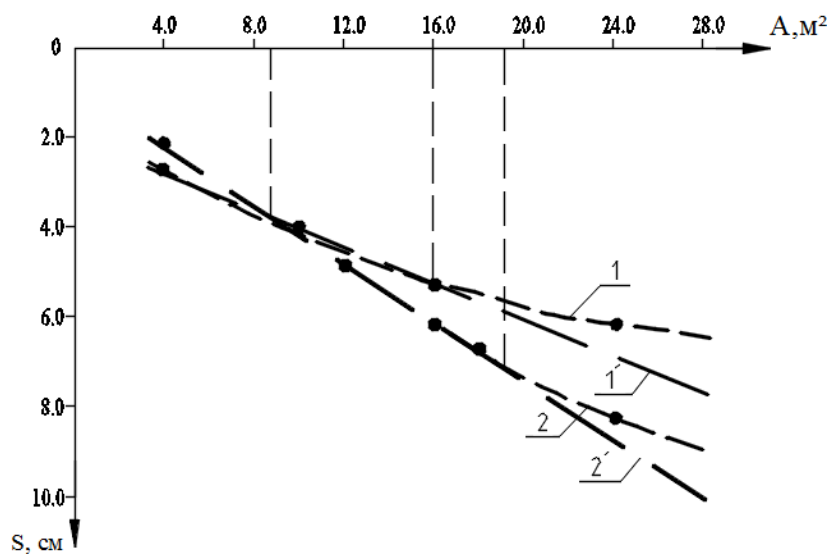

Рисунок 4 - Влияние коэфффициента $\eta=\frac{l}{b}$ на величину осадки фундамента различной ширины

Если ширина фундамента $b=2,0$ м и коэфффициент $\eta=2,5$, то такую же осадку будут иметь фундаменты шириной до 3,0 м, но с коэффициентами отношения сторон до 1,0.

Таким образом очевидно, что выравнивание неравномерных осадок фундаментов следует рассматривать только в определенных диапазонах изменения их ширины и при соответствующих значениях коэфффициента отношения сторон.

\section{Заключение}

При постоянном давлении осадки квадратных (круглых) фундаментов меньше в сравнении с ленточными при прочих равных условиях. При переходе от квадратных и круглых фундаментов к прямоугольным и ленточным и росте коэффициента отношения сторон увеличивается возможность поперечного вытеснения грунта из-под фундамента и соответственно больших осадок.

Осадки прямоугольных фундаментов практически в любом диапазоне изменения коэфффициента отношения сторон всегда находятся по своей величине между осадками квадратных (круглых) и ленточных фундаментов. Увеличение размеров (площади) квадратных и круглых фундаментов в несколько раз (например, в 4 раза) уменьшает их осадку практически пропорционально изменению размеров стороны или диаметра (в 2 раза). Увеличение ширины ленточных фундаментов неэффективно, ибо уменьшение осадки в этом случае незначительно.

Для выравнивания осадок фундаментов зданий и сооружений целесообразно применение фундаментов квадратной (круглой) формы, менее эффективно - прямоугольных, с коэффрициента отношения сторон $\eta$ не превышающем 1,2.

\section{Список цитированных источников}

1. Гольштейн, М. Н. Расчеты осадок и прочности оснований зданий и сооружений / М. Н. Гольштейн, С. Г. Кушнер, М.И.Шевченко. Киев : Будівельник, 1977. - 208 с.

2. Польшин, Д. Е. Влияние площади и формы фундамента на его осадку / Д. Е. Польшин // М., Стройиндустрия, № 3. - 1937. C. 23-29.

3. Хакимов, Х. Р. Экспериментальные исследования деформаций основания / Х. Р. Хакимов // Гидроэнергетическое строительство, № 9, М., 1939. - С. 16-24.

4. Цытович, Н. А. Механика грунтов / Н. А. Цытович // Высшая школа. - М., 1979. - 272 c.

5. Шведовский, П. В. Выбор оптимальных решений в строительстве / П. В. Шведовский, А. М. Мальцев, Т. И. Мальцева. - М., ЦНИИПсельстрой, 1990. - 396 с.

6. Muhs, $\mathrm{H}$ Ergebnisse von Probebelastungen auf großen Lastflächen zur Ermittlung der Bruchlast von Sand / H. Muchs // (Bericht Versuche mit ausmittigem Lastangriff). - 1961. - S. 59-98.

\section{References}

1. Gol'shtejn, M. N. Raschety osadok i prochnosti osnovanij zdanij i sooruzhenij / M. N. Gol'shtejn, S. G. Kushner, M. I. Shevchenko. Kiev : Budivel'nik, 1977. - 208 s.

2. Pol'shin, D. E. Vliyanie ploshchadi i formy fundamenta na ego osadku. / D. E. Pol'shin // M., Strojindustriya, № 3. - 1937. - S. 23-29.

3. Hakimov, H. R. Eksperimental'nye issledovaniya defor-macij osnovaniya / H. R. Hakimov // Gidroenergeticheskoe stroitel'stvo, № 9, M., 1939. - S. 16-24.

4. Cytovich, N. A. Mekhanika gruntov / N.A. Cytovich // Vysshaya shkola. - M., 1979. - 272 s.

5. Shvedovskij, P. V. Vybor optimal'nyh reshenij v stroi-tel'stve / P. V. Shvedovskij, A. M. Mal'cev, T. I. Mal'ceva. - M., CNIIPsel'stroj, 1990. - $396 \mathrm{~s}$.

6. Muhs, $\mathrm{H}$ Ergebnisse von Probebelastungen auf gro־en Last-flächen zur Ermittlung der Bruchlast von Sand / H. Muchs // (Bericht Versuche mit ausmittigem Lastangriff). - 1961. - S. 59-98.

Материал поступил в редакцию 06.05.2021 